\title{
Die Eier legende Wollmilchsau?
}

\section{Liebe Leserinnen und Leser,}

wir hoffen nicht, dass Sie des Schlagworts Digitalisierung schon überdrüssig sind - man kann heute ja kaum noch etwas zum Controlling lesen (oder schreiben!), in dem der Bezug dazu fehlt. Nicht zu Unrecht: Die Digitalisierung läutet eine neue Ära von Management und Controlling ein, die auch erhebliche Konsequenzen für die von Controllern benötigten Fähigkeiten hat.

Dennoch ist sie nicht die erste grundlegende Veränderung im Controlling: Schaut man auf die Entstehung des Berufsstandes zurück, so hatten Controller vor gut 50 Jahren alle Hände voll damit zu tun, führungsrelevante Informationen zu erfassen und bereitzustellen, um damit die physische Basis zu schaffen, die vorhandenen instrumentellen Konzepte in der Praxis anwendbar zu machen. Ihr Platz war dominant „im Maschinenraum“, sie durften sich auch für Programmierungsaufgaben nicht zu schade sein.

Spätestens mit der Einführung von ERP-Systemen wurden sie von der Datenbeschaffung weitgehend entlastet. Sie konnten sich stärker darauf konzentrieren zu überlegen, wie die vielfältigen Informationen verwendet werden sollten. Dies erforderte einen engeren Kontakt mit den Managern. Controller brauchten hierzu zum einen genauere Geschäftskenntnisse, um die Manager und ihre Probleme zu verstehen. Zum anderen mussten sie ihr Mindset ändern und auf die Manager zugehen: Hier ist die altbekannte Forderung von Albrecht Deyhle nach dem „Zahlenverkäufer“ zu verorten. Aus dem Registrator wurde der Navigator mit einem deutlich breiteren Skillset.

Verbunden mit dem Begriff des Business Partners ist dann ein weiterer Entwicklungsschritt vollzogen worden, der die Zusammenarbeit mit den Managern ganz in den Fokus stellt und das Anforderungssniveau für die Controller noch weiter nach oben schraubt. Ein aktives „Mit-Managen“ erfordert noch intimere Geschäftskenntnisse und ein noch besseres Verstehen der Manager. Die zunehmende Verhaltensorientierung im Controlling ist dementsprechend alles andere als ein Zufall.

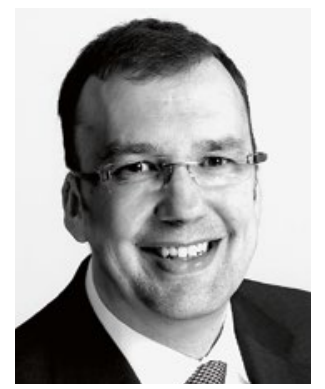

Utz Schäffer

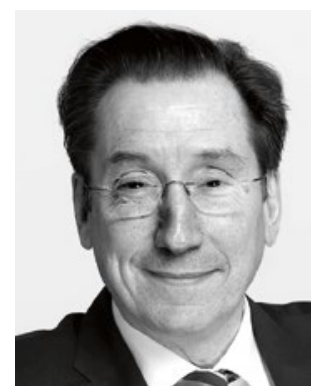

Jürgen Weber
Und jetzt auch noch Digitalisierung! Controller müssen lernen, digitale Geschäftsmodelle zu verstehen, sie müssen sich neue Instrumente aneignen und ihr Mindset erneut verändern: Wer keine Abweichungen mag, dem wird es schwerfallen, sich mit einem „Fail fast" anzufreunden.

Wird der Controller-Bereich damit zwingend überfordert? Wir glauben nein. Wird jeder Controller den neuen Anforderungen genügen können? Auch hier glauben wir nein. Wir brauchen daher mehr Offenheit gegenüber neuen Rollenbildern - unser Beitrag in diesem Heft geht darauf ein - und eine Team-Perspektive! Nicht jeder muss im Controlling alles können. Es geht um eine Art von „Schwarmfähigkeit“. Sonst sind wir wirklich schnell bei der Eier legenden Wollmilchsau!

Viel Spaß bei der Lektüre wünschen Ihnen

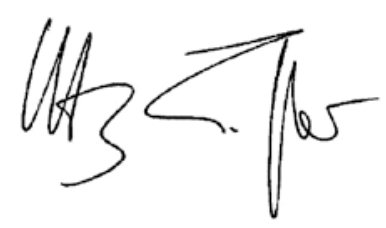

Utz Schäffer

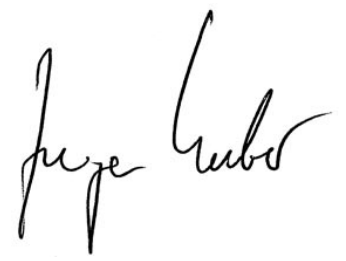

Jürgen Weber 